\title{
Pkd Proteins Team Up to Tell Cilia Which Way to Go
}

\author{
Domenico F. Galati \\ Department of Cell and Developmental Biology, University of Colorado Denver-Anschutz Medical Campus, Aurora, Colorado 80045-2537 \\ Review of Ohata et al.
}

Cilia are membrane-ensheathed microtubule bundles that protrude from nearly every cell in the nervous system, including neurons, glia, and ventricular ependymal cells (E-cells). Although their role was initially obscure, it is becoming increasingly clear that nervous system cilia are essential for multiple facets of brain development (Guemez-Gamboa et al., 2014). This is because cilia perform two highly specialized functions. First, the whip-like beating of motile cilia, which are exclusively found on the surface of E-cells, propels CSF through the brain's ventricles. Disrupting motile cilia abrogates cilia-generated CSF flow, leading to hydrocephalus. Second, virtually all neurons, glia, and E-cell precursors harbor a single primary cilium that transduces critical developmental signaling molecules, including Sonic hedgehog and Wnt. Perturbation of primary cilia impairs the migration and differentiation of neurons, glia, and E-cells, leading to gross brain malformations and cognitive impairments.

To function properly, cilia must be positioned appropriately relative to the plane of the cell sheet. This is called planar

Received Oct. 6, 2015; revised Nov. 10, 2015; accepted Nov. 17, 2015.

D.F.G. is supported by the National Institute of General Medical Sciences Grant GM0099820, the Boettcher Webb-Waring Foundation, and the Pew Biomedical Scholars Program to Dr. Chad G. Pearson. I thank Dr. Chad G. Pearson and members of the Pearson lab for helpful discussions about cilia and basal body polarity.

The author declares no competing financial interests.

Correspondence should be addressed to Domenico F. Galati, Department of Cell and Developmental Biology, University of Colorado Denver-Anschutz Medical Campus, Aurora, C0 80045-2537. E-mail: domenico.galati@ucdenver.edu.

DOI:10.1523/JNEUROSCI.3684-15.2016

Copyright $\odot 2016$ the authors $\quad 0270-6474 / 16 / 360643-03 \$ 15.00 / 0$ cell polarity (PCP) and in the case of E-cells, which develop dense multiciliated patches, the arrangement of cilia exhibits two types of planar polarization (Mirzadeh et al., 2010). First, the patch of cilia is offset from the center of the E-cell apical surface toward the direction of CSF flow; this is called translational polarity. Second, cilia within the patch are oriented so their force-generating power stroke beats in line with CSF flow; this is called rotational polarity. Interfering with E-cell cilia polarity alters the magnitude and/or the direction of cilia-generated hydrodynamic force, which ultimately leads to CSF accumulation and hydrocephalus (Boutin et al., 2014). Cilia polarity is clearly important for the development and function of the brain's ventricular system. However, the mechanisms that establish cilia polarity within the ventricles are poorly understood.

E-cells arise from a subset of polarized radial glia cells (RGCs) that line the early postnatal ventricular epithelium (Spassky et al., 2005). Before differentiation, ventricular RGCs contain a single primary cilium that projects from the apical surface into the ventricular cavity. When RGCs commit to E-cell differentiation, they undergo massive ciliogenesis at their apical membrane, and the resulting multiciliated patch acquires both translational and rotational polarity. Previous studies demonstrated that RGC primary cilia are required for the polarization of E-cell motile cilia upon differentiation. Specifically, RGCs that lack primary cilia differentiate into E-cells with a normal density of basal bodies, which are the structures that nu- cleate motile cilia. However, the translational polarity of these basal bodies is disrupted, as evidenced by a more centralized positioning of the basal body patch on the E-cell apical surface (Mirzadeh et al., 2010). Although this prior study demonstrated that RGC primary cilia are important for E-cell polarity, cilia contain a plethora of signaling molecules, so more subtle perturbations are required to understand the mechanisms that influence E-cell PCP.

One pair of molecules that affect PCP is the ciliary mechanosensory proteins polycystic kidney disease $1(\mathrm{Pkd} 1)$ and $\mathrm{Pkd}$ 2. $\mathrm{Pkd} 1$ and $\mathrm{Pkd} 2$ are multipass transmembrane proteins that localize to both ciliary and plasma membranes (Retailleau and Duprat, 2014). In the kidney, Pkd1 and $\mathrm{Pkd} 2$ are thought to form a ciliary mechanosensory complex that translates fluid flow into second messenger signals via $\mathrm{Pkd} 2$-mediated $\mathrm{Ca}^{2+}$ conductance. Pkd1 loss-of-function disrupts oriented cell division and the elongation of polarized extensions in the kidney (Luyten et al., 2010; Castelli et al., 2013), and brainspecific $\mathrm{Pkd} 1$ deletion causes hydrocephalus (Wodarczyk et al., 2009). Thus, Pkd proteins are strong candidates to influence PCP in the ventricular epithelium.

Based on these studies, Ohata et al. (2015) hypothesized that Pkd1 and Pkd2 may sense early CSF flow and translate it into ventricular planar polarity. To test this hypothesis, they first examined Pkd expression and $\mathrm{Pkd}$ function in the developing ventricles. By postnatal day 0 (P0), the ventricular epithelium is lined with RGCs. Pkd1 and Pkd2 were both ex- 
pressed in RGCs at this developmental stage, and they colocalized in discrete clusters along the RGC's primary cilium as well as in patches on the apical membrane. Moreover, the ciliary localization of $\mathrm{Pkd} 2$ depended upon the ciliary localization of $\mathrm{Pkd} 1$, suggesting that $\mathrm{Pkd} 1$ and $\mathrm{Pkd} 2$ form a complex within the RGC cilium.

To understand $\mathrm{Pkd}$ function, Ohata et al. (2015) conditionally ablated Pkd1 and $\mathrm{Pkd} 2$ in the embryonic nervous system using Nestin-cre, which drives recombination in neuron and glia precursors, including the RGCs that give rise to E-cells. At a gross level, brain ventricles developed normally in these animals. By P21, rosettes were abundant and there were no differences in the number of cilia per E-cell, cilia length, or the frequency of cilia beating. However, the transmembrane protein Vangl2, which contributes to the establishment of PCP, was disrupted in E-cells. Specifically, Vangl2 levels were significantly decreased, and the Vangl2 that was present was diffusely distributed across the E-cell surface rather than being translational polarized along the edge of the E-cell. Thus, Pkd proteins are not required for the gross development of the ventricular epithelium or for E-cell cilia formation, but they are important for setting up the asymmetric localization of Vangl2.

Vangl2 directs multiple aspects of cilia polarity in vertebrates (Borovina et al., 2010), and this led Ohata et al. (2015) to examine ventricular cilia polarity in both RGCs and differentiated E-cells. In Pkd mutants at $\mathrm{P} 0$, the translational polarity of RGC primary cilia was disrupted, as evidenced by altered displacement of the primary cilium's nucleating structure called the basal body. Moreover, the polarity defects persisted after RGC differentiation: by P21, E-cell cilia patches were mislocalized and the rotational polarities of cilia within the patch were oriented in random directions rather than uniformly aligned. Importantly, although cilia beat frequency was normal on Pkd mutant E-cells, the ability of Pkd mutant ventricles to generate hydrodynamic force was compromised, which suggests that altered cilia polarity contributes to hydrocephaly in Pkd1 mutant animals (Wodarczyk et al., 2009).

To determine whether Pkd proteins and Vangl2 function in the same cilia polarity pathway, Ohata et al. (2015) examined Pkd1 and Vangl2 double heterozygous mutant animals. According to classical genetic principles, if $\mathrm{Pkd} 1$ and Vangl2 function in parallel pathways, then heterozygous loss-of-function mutations in both genes should have an additive effect on cilia polarity. However, double heterozygous mutations had a synergistic effect on both translational and rotational cilia polarity, which indicates that Pkd1 and Vangl2 likely function in the same pathway to control E-cell cilia polarity.

Ohata et al. (2015) provide strong in vivo evidence that $\mathrm{Pkd}$ proteins contribute to cilia polarity throughout ventricular development, but whether this requires ventricular mechanosensation by $\mathrm{Pkd} 1$ and $\mathrm{Pkd} 2$, as occurs in kidney, warrants further investigation. Most models of ciliary mechanotransduction rely on $\mathrm{Pkd} 2$ 's ability to induce $\mathrm{Ca}^{2+}$-induced $\mathrm{Ca}^{2+}$ release by acting as a ciliary $\mathrm{Ca}^{2+}$ channel (Nauli et al., 2003). However, recent patch-clamp experiments demonstrated that $\mathrm{Pkd} 2$ is neither necessary nor sufficient to conduct a ciliary $\mathrm{Ca}^{2+}$ current (DeCaen et al., 2013). Therefore, if Pkd2 functions as a mechanosensitive ciliary $\mathrm{Ca}^{2+}$ channel, it may require unknown interacting partners or conditions that are unique to the RGC primary cilium. An alternative possibility is that mechanosensation by $\mathrm{Pkd} 1 / \mathrm{Pkd} 2$ activates the $\mathrm{Pkd} 2$ family member Pkd2l1, which is a confirmed high-conductance ciliary $\mathrm{Ca}^{2+}$ channel (DeCaen et al., 2013), although it is unknown whether $\mathrm{Pkd} 2 \mathrm{ll}$ is present within RGC primary cilia. Regardless of Pkd's involvement in RGC mechanosensation, it will be important to directly test the role of passive CSF flow in setting up ventricular cilia polarity. One clear prediction from Ohata et al. (2015) is that manipulating CSF flow in the early ventricles should influence cilia polarity on differentiated E-cells. Importantly, similar flow experiments have been performed in the developing Xenopus embryo with great success (Mitchell et al., 2007).

Since Pkd proteins and Vangl2 function in the same cilia polarity pathway, it will be necessary to tease apart the functional interaction between Pkd and Vangl2. In multiciliated airway epithelial cells, planar polarized cytoplasmic microtubules are required for the asymmetric distribution of Vangl2 (Vladar et al., 2012). Cytoplasmic microtubules are organized by the centrosome, and in RGCs, the centrosome is located at the base of the primary cilium. Thus, one intriguing possibility is that RGCs translate ciliary mechanosensation into polarized microtubules via the centrosome, which allows polarized Vangl2 localization. This model could be tested by determining whether
CSF flow affects cytoplasmic microtubule organization in the ventricles before E-cell differentiation. Since PCP is established in the nervous system before P0 (Goodrich, 2008), which is when Ohata et al. (2015) began their analyses, it is also important to temporally resolve the interaction between Pkd proteins and Vangl2. Specifically, Nestin, Vangl2, $\mathrm{Pkd} 1$, and $\mathrm{Pkd} 2$ expression can all be detected in the developing nervous system as early as E11.5, while the RGCs that give rise to most E-cells are born around E16 (Spassky et al., 2005). Thus, it is necessary to examine asymmetries in the cell divisions that precede the birth of ventricular RGCs because translational polarity defects in $\mathrm{Pkd}$ mutant RGCs could reflect asymmetries inherited from prior cell divisions rather than defective flow sensing.

In conclusion, Ohata et al. (2015) convincingly demonstrate that both $\mathrm{Pkd} 1$ and $\mathrm{Pkd} 2$ play essential roles in setting up the intricate polarity of multiciliated E-cells in the ventricular epithelium. Through their detailed analyses of cilia polarity and CSF flow in Pkd mutants, the authors have identified a novel pathway that may allow early passive CSF flow to be converted into the much stronger flow that is characteristic of the mature ventricular system. These results represent a fundamental discovery in our basic understanding of cilia-derived mechanosensation, as well as a critical demonstration of the primary cilium's importance as a key regulator of nervous system development.

\section{References}

Borovina A, Superina S, Voskas D, Ciruna B (2010) Vangl2 directs the posterior tilting and asymmetric localization of motile primary cilia. Nat Cell Biol 12:407-412. CrossRef Medline

Boutin C, Labedan P, Dimidschstein J, Richard F, Cremer H, Andre P, Yang Y, Montcouquiol M, Goffinet AM, Tissir F (2014) A dual role for planar cell polarity genes in ciliated cells. Proc Natl Acad Sci U S A 111:E3129-E3138. CrossRef Medline

Castelli M, Boca M, Chiaravalli M, Ramalingam H, Rowe I, Distefano G, Carroll T, Boletta A (2013) Polycystin-1 binds Par3/aPKC and controls convergent extension during renal tubular morphogenesis. Nat Commun 4:2658. CrossRef Medline

DeCaen PG, Delling M, Vien TN, Clapham DE (2013) Direct recording and molecular identification of the calcium channel of primary cilia. Nature 504:315-318. CrossRef Medline

Goodrich LV (2008) The plane facts of PCP in the CNS. Neuron 60:9-16. CrossRef Medline

Guemez-Gamboa A, Coufal NG, Gleeson JG (2014) Primary cilia in the developing and mature brain. Neuron 82:511-521. CrossRef Medline 
Luyten A, Su X, Gondela S, Chen Y, Rompani S, Takakura A, Zhou J (2010) Aberrant regulation of planar cell polarity in polycystic kidney disease. J Am Soc Nephrol 21:1521-1532. CrossRef Medline

Mirzadeh Z, Han YG, Soriano-Navarro M, García-Verdugo JM, Alvarez-Buylla A (2010) Cilia organize ependymal planar polarity. J Neurosci 30:2600-2610. CrossRef Medline

Mitchell B, Jacobs R, Li J, Chien S, Kintner C (2007) A positive feedback mechanism governs the polarity and motion of motile cilia. Nature 447:97-101. CrossRef Medline

Nauli SM, Alenghat FJ, Luo Y, Williams E, Vassilev P, Li X, Elia AE, Lu W, Brown EM, Quinn
SJ, Ingber DE, Zhou J (2003) Polycystins 1 and 2 mediate mechanosensation in the primary cilium of kidney cells. Nat Genet 33: 129-137. CrossRef Medline

Ohata S, Herranz-Pérez V, Nakatani J, Boletta A, García-Verdugo JM, Álvarez-Buylla A (2015) Mechanosensory genes $\mathrm{Pkd} 1$ and $\mathrm{Pkd} 2$ contribute to the planar polarization of brain ventricular epithelium. J Neurosci 35:1115311168. CrossRef Medline

Retailleau K, Duprat F (2014) Polycystins and partners: proposed role in mechanosensitivity. J Physiol 592:2453-2471. CrossRef Medline

Spassky N, Merkle FT, Flames N, Tramontin
AD, García-Verdugo JM, Alvarez-Buylla A (2005) Adult ependymal cells are postmitotic and are derived from radial glial cells during embryogenesis. J Neurosci 25:1018. CrossRef Medline

Vladar EK, Bayly RD, Sangoram AM, Scott MP Axelrod JD (2012) Microtubules enable the planar cell polarity of airway cilia. Curr Biol 22:2203-2212. CrossRef Medline

Wodarczyk C, Rowe I, Chiaravalli M, Pema M, Qian F, Boletta A (2009) A novel mouse model reveals that polycystin-1 deficiency in ependyma and choroid plexus results in dysfunctional cilia and hydrocephalus. PloS One 4:e7137. CrossRef Medline 